\title{
Étude des potentialités des systèmes d'application contrôlée des gouttes (CDA) pour les traitements phytosanitaires en céréaliculture (synthèse bibliographique)
}

\author{
Sofiene Ouled Taleb Salah ${ }^{(1,2)}$, Nicolas De Cock ${ }^{(2)}$, Mathieu Massinon ${ }^{(2)}$, \\ Bruno Schiffers ${ }^{(3)}$, Stéphane Dorbolo ${ }^{(4)}$, Frédéric Lebeau ${ }^{(2)}$ \\ (1) Université de Liège - Gembloux Agro-Bio Tech. TERRA. AgricultureIsLife. Passage des Déportés, 2. BE-5030 Gembloux \\ (Belgique). \\ (2) Université de Liège - Gembloux Agro-Bio Tech. Biosystems Engineering. Axe Agriculture de Précision. Passage des \\ Déportés, 2. BE-5030 Gembloux (Belgique).E-mail : f.lebeau@ulg.ac.be \\ (3) Université de Liège - Gembloux Agro-Bio Tech. Agrobiochem. Laboratoire de Phytopharmacie. Passage des Déportés, 2. \\ BE-5030 Gembloux (Belgique). \\ (4) Université de Liège. GRASP. Institut de Physique. Bâtiment 5a. Sart-Tilman. BE-4000 Liège (Belgique).
}

Reçu le 1 avril 2015, accepté le 12 novembre 2015.

Introduction. L'application contrôlée des gouttes (CDA) présente le double avantage de réduire à la fois l'étendue de la distribution des tailles des gouttes et le volume appliqué par rapport aux buses hydrauliques conventionnelles.

Littérature. Cette revue investigue la potentialité de la CDA par rapport aux buses hydrauliques dans le cadre du désherbage précoce des adventices (2-3 feuilles). En termes de rétention, les études reliant les caractéristiques des gouttes (taille, vitesse et direction) et l'architecture, la densité et la mouillabilité du feuillage, en fonction de la formulation de bouillies, ont été abordées. Ces études montrent que des gouttes de $300 \mu \mathrm{m}$ avec un adjuvant approprié et des jets émis $60^{\circ}$ vers l'avant par rapport à la verticale contribuent à l'augmentation de la rétention sur les cibles problématiques à port dressé. L'effet des réductions des volumes d'application pour ces deux techniques est étudié sur base de la moyenne, du coefficient de variation et du taux de couverture des dépôts. Néanmoins, les résultats de ces études ne peuvent être généralisés du fait des différences de réglages opératoires entre les deux techniques d'application. Quant à la dérive, les atomiseurs CDA orientés horizontalement présentent un potentiel de dérive plus important que les buses hydrauliques pour lesquelles le spray est orienté vers le bas. La dérive augmente avec la vitesse du vent et diminue lorsque le diamètre médian volumétrique $\left(\mathrm{DV}_{50}\right)$ augmente.

Conclusions. De nouveaux réglages des atomiseurs de la CDA en termes de $\mathrm{DV}_{50}$ et de direction du jet peuvent répondre aux défis de l'agriculture de précision. Les pistes d'innovations proposées sont d'une part une amélioration de la résistance à l'usure des dents situées en bordure des disques et, d'autre part, une distribution granulométrique plus étroite.

Mots-clés. Application des pesticides, pulvérisateur, buse, adjuvant, rétention, dérive.

\section{Study of the potential for the use of controlled droplet application (CDA) upon cereal crops. A review}

Introduction. Controlled droplet application (CDA) has the advantage both of the reduction in the span of droplet size distribution and in the volume of liquid applied, in comparison with conventional hydraulic nozzles.

Literature. This review investigates the potential for the use of CDA relative to hydraulic nozzles within the early stages of weed control (2-3 leaves). The studies reviewed examine spray retention, linking the droplet characteristics (size, velocity and direction) of spray liquids with the architecture, density and wettability of the foliage. These studies show that the use of droplets of $300 \mu \mathrm{m}$, combined with an appropriate adjuvant and applied with a $60^{\circ}$ forward-angled spray, led to the enhanced depositing of liquids on upright surfaces. Assessment of the reduced volumes applied by both application techniques was based on the mean, the variation coefficient and the deposition coverage. However, these results cannot be generalized, due to the different operative settings in which the spray application techniques were employed. Regarding the risk of drift to the side, the drift potential of droplets generated horizontally by CDA nozzles is greater than that of the conventional technique with a downward orientation of spray. The drift potential increases as the wind velocity increases and the volume median diameter $\left(\mathrm{DV}_{50}\right)$ decreases. 
Conclusions. New settings of CDA nozzles in terms of $\mathrm{DV}_{50}$ and the direction of spray may address the challenge of precision agriculture. Innovative ways to apply pesticides, based on CDA, must be explored in order to avoid the sensitivity to wear of the teeth in spray applicators and to obtain a narrower droplet-size distribution.

Keywords. Pesticide application, sprayers, nozzles, adjuvant, spray retention, spray drift.

\section{INTRODUCTION}

La protection des cultures céréalières est essentiellement réalisée par l'application de produits phytosanitaires. La pulvérisation consiste à fragmenter une bouillie agricole en une multitude de gouttes. Cette fragmentation est réalisée à l'aide de différents actionneurs dédiés. Le jet ainsi formé contient des gouttes présentant des tailles et des vitesses différentes selon le type d'actionneur utilisé, les réglages utilisés (pression, débit) et les propriétés physicochimiques de la bouillie (tension de surface, viscosité, densité). La classification de la qualité du jet se base sur la taille des gouttes en séparant les jets en sept classes distinctes : très fin / fin / moyen / gros / très gros / extrêmement gros / ultra gros (Doble et al., 1985 ; Southcombe et al., 1997). La distribution granulométrique d'un jet peut être caractérisée de manière quantitative à l'aide des paramètres de positions : $\mathrm{DV}_{10}, \mathrm{DV}_{50}$ et $\mathrm{DV}_{90}$ désignant respectivement les diamètres de gouttes auxquels $10 \%$, $50 \%$ et $90 \%$ du volume total du jet sont inférieurs. L'uniformité de la distribution granulométrique est estimée par un indice adimensionnel appelé facteur d'étalement relatif (relative span factor, RSF) défini comme suit : $\operatorname{span}=\left(\mathrm{DV}_{90}-\mathrm{DV}_{10}\right) / \mathrm{DV}_{50}$. Plus la valeur du span est élevée, plus la distribution des tailles des gouttes est étendue et, par conséquent, l'uniformité du spectre faible. La pulvérisation en céréaliculture utilise principalement des buses hydrauliques. Cet actionneur force le passage de la bouillie sous pression au travers d'orifices calibrés générant une nappe liquide qui se rompt en gouttes sous l'action d'instabilités (Elbanna et al., 1984). Le jet généré possède une distribution granulométrique présentant un span aux alentours de 1 (il varie de 0,8 à 1,3) (Mathews, 1992 ; Qi et al., 2008).

La tendance actuelle est à la réduction des volumes appliqués pour des raisons économiques et environnementales. Ces réductions de volumes appliqués sont rendues possibles par la diminution du calibre de la buse hydraulique, de la pression de pulvérisation et par l'augmentation de la vitesse d'avancement lors du traitement. Ces modifications de la technique d'application affectent drastiquement les caractéristiques du jet notamment le nombre, la taille et la vitesse des gouttes (Nuyttens et al., 2009) et influent, par conséquent, sur l'efficience du traitement. À titre d'exemple, les plus fines gouttes, même si elles assurent une bonne rétention (quantité du produit capturé par le feuillage) (Skuterud et al., 1988 ; Knoche, 1994), sont sensibles à la dérive (transport de gouttes hors de la zone cible) (Matthews, 1992). Les gouttes de tailles plus importantes, en fonction de leur énergie cinétique, de la tension de surface ainsi que de la mouillabilité de la surface de la cible, ont tendance à rebondir ou à se fragmenter lors de l'impact (Massinon et al., 2012b ; Boukhalfa et al., 2014), réduisant ainsi les dépôts sur le feuillage (Wolf et al., 2000). Par ailleurs, ces grosses gouttes, caractéristiques des buses anti-dérives qui sont utilisées à basse pression ou des buses à induction d'air, ont une sensibilité réduite à la dérive.

En vue de limiter les problèmes inhérents à l'utilisation des buses hydrauliques (à jets plats et à induction d'air), la technique d'application contrôlée des gouttes (controlled droplet application, CDA), notamment par le biais d'actionneurs appelés atomiseurs rotatifs, a été développée. Ces atomiseurs utilisent l'énergie centrifuge pour fragmenter une nappe liquide en gouttes (Frost, 1981). Cette pratique présente le double avantage de réduire la dispersion du spectre des gouttes, attesté par une valeur du span autour de 0,5 , et de diminuer le volume à l'hectare appliqué (Derksen et al., 1986 ; Matthews, 1992). En outre, la taille et la vitesse des gouttes peuvent être adaptées en fonction du traitement envisagé grâce à une gamme de vitesses de rotations et de débits (Walton et al., 1949 ; Bode et al., 1983 ; Clayton, 1992 ; Matthews, 1992 ; Clayton et al., 1993 ; Holland et al., 1997 ; Zainoun et al., 2004). Le diamètre des gouttes produites par cet actionneur peut être estimé approximativement à partir de l'équation suivante (Walton et al., 1949) :

$$
\mathrm{d}=(\mathrm{K} \cdot 1) / \mathrm{w} \cdot(\sigma / \mathrm{D} \cdot 1)^{1 / 2}
$$

avec $\mathrm{d}$ : diamètre de la goutte $(\mu \mathrm{m}), \mathrm{w}$ : vitesse angulaire $(\mathrm{rad} / \mathrm{s}), \mathrm{D}:$ diamètre du disque $(\mathrm{mm}), \sigma$ : tension de surface du liquide $\left(\mathrm{mN} \cdot \mathrm{m}^{-1}\right), 1:$ densité du liquide $\left(\mathrm{g} \cdot \mathrm{cm}^{3}\right), \mathrm{K}:$ constante déterminée expérimentalement. Malgré les réticences des agriculteurs vis-à-vis de ces actionneurs pour des raisons de couts (Clayton, 1992), leur utilisation permettrait théoriquement de satisfaire les objectifs de réduction des volumes appliqués et d'augmentation de l'efficience des traitements.

Néanmoins en cas de réglage inadéquat de l'actionneur, la distribution granulométrique de ces atomiseurs rotatifs peut être caractérisée par une double distribution bimodale du jet avec une première distribution de gouttes fines (inférieures à $100 \mu \mathrm{m}$ ) et une distribution principale plus large qui est relativement peu étalée (Walton et al., 1949 ; Bode et al., 1972). Ce type de distribution apparait lorsque la rupture en 
bordure des atomiseurs rotatifs est imparfaite, le nuage de fines gouttes correspond alors aux gouttes satellites. En effet, elles se forment à partir de la queue (fil) reliant la gouttelette principale au reste du ligament liquide (Dombrowski et al., 1974). Par conséquent, un réglage adéquat de l'actionneur est crucial en vue de produire un jet avec une granulométrie resserrée.

La présente étude bibliographique vise à investiguer le potentiel de la CDA à relever les défis agronomiques en termes de maximisation de dépôts sur la cible et de limitation de dérive dans le cadre du désherbage foliaire précoce des adventices céréaliers à surface superhydrophobe (stade 2-3 feuilles), notamment le vulpin des champs, Alopecurus myosuroides Huds, par rapport aux buses hydrauliques. Une représentation systématique des composantes impliquées dans la potentialité de la pulvérisation, y compris la CDA, a été établie (Figure 1). Cette dernière comporte trois composantes majeures: les réglages opératoires de l'atomiseur, la cible et les conditions externes. Les interactions entre ces composantes et les caractéristiques de chaque composante sont respectivement indiquées dans des cases en pointillés et à trait plein. À travers cette représentation, l'accent est mis sur les combinaisons possibles existantes entre la technique d'application en termes de tailles, direction et vitesse d'éjection des gouttes et les formulations de la bouillie en fonction de la nature de la cible à traiter et du vent.

Cette revue constitue un outil d'aide à la mise au point des bonnes pratiques de pulvérisation agricole basées sur l'utilisation rationnelle et sécurisée de produits phytosanitaires. Elle introduit également de nouvelles approches et pistes innovantes de contrôle du processus des traitements phytosanitaires.

Ce document est scindé en deux parties majeures traitant l'impact de la CDA sur l'aspect rétention d'une part et sur l'aspect dérive d'autre part, en fonction des paramètres physicochimiques mis en jeu.

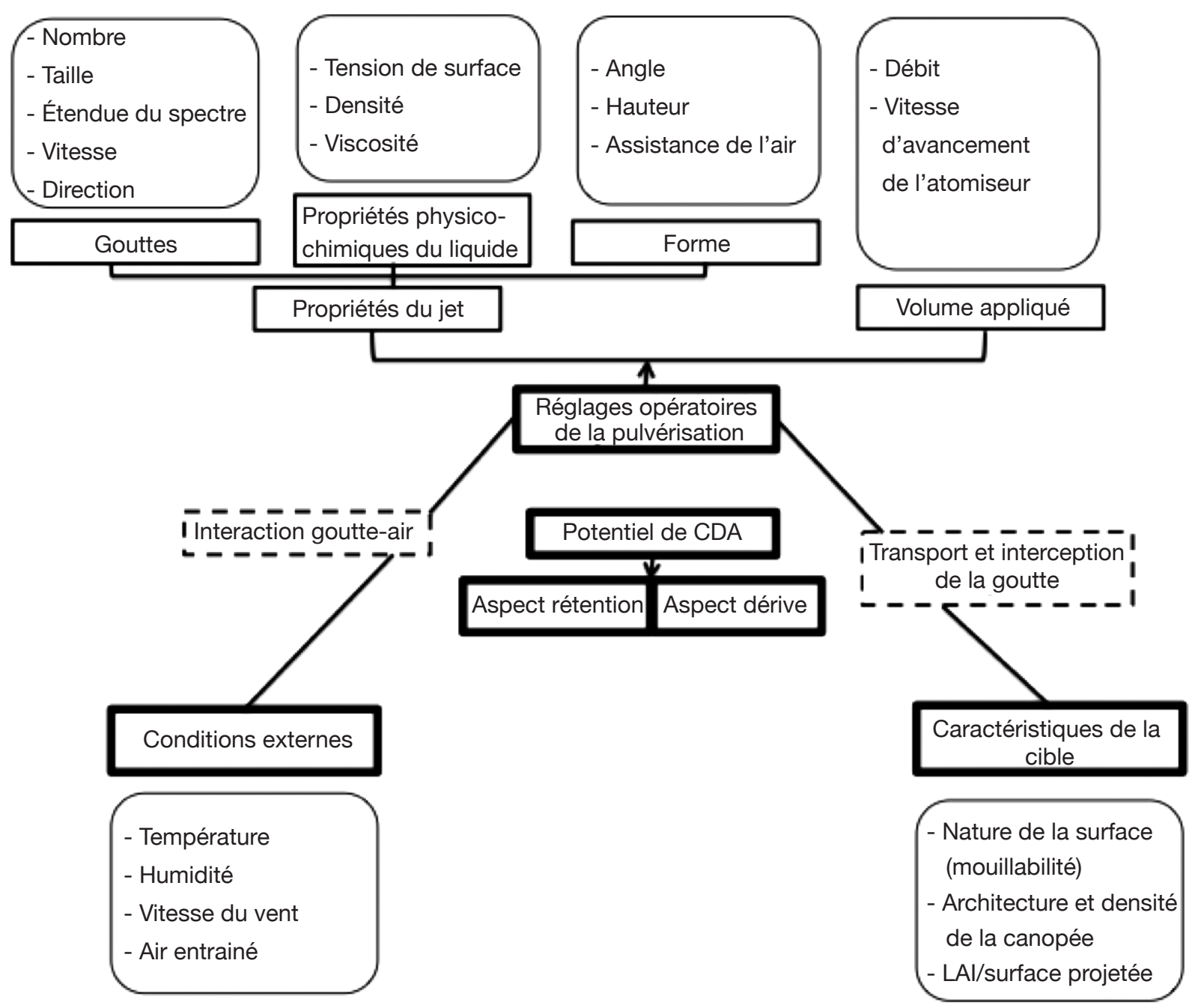

Figure 1. Représentation systématique des composantes impliquées dans l'étude de la potentialité de la CDA - Systemic representation of components involved in the assessment of CDA potential. 


\section{ASPECT RÉTENTION}

\subsection{Propriétés de l'adventice à traiter}

Les propriétés des adventices figurent parmi les paramètres influençant la rétention des produits phytosanitaires. À l'échelle microscopique, la mouillabilité de la surface et la tension de surface de la bouillie conditionnent le comportement des gouttes au niveau de la surface. À l'échelle macroscopique, la densité de la canopée et l'architecture de la plante (surface et orientation foliaire) sont les facteurs déterminants dans ce processus.

Échelle microscopique. La mouillabilité caractérise l'aptitude d'une surface foliaire à se laisser mouiller par un liquide. La mouillabilité dépend de l'espèce, de la variété, du stade de croissance, de la position de la feuille, des conditions de culture et des facteurs environnementaux (Butler Ellis et al., 2004 ; Taylor, 2011; Massinon et al., 2012b). En fonction du degré de mouillabilité, on distingue les surfaces faciles à mouiller et celles difficiles à mouiller (Zabkiewicz, 2007). Cette caractéristique de la surface est déterminée par la mesure d'un angle de contact d'équilibre décrivant l'interface goutte/surface/air. Plus cet angle de contact est élevé, plus la mouillabilité de la surface diminue et la surface est qualifiée dès lors d'hydrophobe voire de super-hydrophobe tel que pour le vulpin des champs. L'hydrophobicité de la surface est souvent compensée par l'ajout d'additifs super-mouillants dans la bouillie et qui favorisent l'étalement de la goutte sur la surface grâce à la réduction de la tension de surface du liquide.

$\mathrm{Au}$ delà de ces considérations, la rétention est également liée au comportement de la goutte lors de l'impact, fortement déterminé par l'énergie d'impact, à la rugosité de la surface et aux propriétés du liquide. Les types d'impacts majeurs sont le rebond, l'adhésion et la fragmentation. L'utilisation de substrats synthétiques super-hydrophobes a permis l'étude des différents paramètres géométriques et chimiques impliqués lors des impacts. Ainsi, une carte décrivant les comportements possibles de la goutte sur ce type de surface en fonction de la rugosité et de la vitesse d'impact a été établie (Rioboo et al., 2008 ; Massinon et al., 2012b). Plus de détails sur la propriété des surfaces et les interactions de la goutte-surface sont disponibles dans la littérature (Massinon et al., 2013 ; Bédiaf et al., 2015). En sus de la mouillabilité de la surface foliaire, l'architecture de la plante constitue un facteur primordial influençant le comportement de la goutte sur celle-ci et affecte la variabilité des dépôts.

Échelle macroscopique. La probabilité d'interception des gouttes constitutives du jet est essentiellement affectée par la densité de la canopée et l'architecture de la plante (Matthews, 1992). La densité de la canopée céréalière augmente avec le stade de croissance de la plante. Ceci confère une bonne interception des gouttes grâce à la surface foliaire développée, caractérisée par un indice de surface foliaire (LAI) élevé. En effet, des essais de laboratoires menés sur une culture dense de blé ont montré que les dépôts ne dépendent ni des propriétés de la bouillie appliquée ni de la nature de la buse hydraulique testée (Butler Ellis et al., 2004) mais sont uniquement conditionnés par la cible. Par contre, dans le cas de densités de végétation faibles, les dépôts sont fortement liés aux propriétés du liquide pour chaque type de buse testée (Butler Ellis et al., 2004). L'orientation du feuillage varie en fonction des stades de croissance et des espèces. L'augmentation de l'inclinaison de la surface foliaire (de l'horizontal à la verticale) réduit la surface verticale projetée qui est disponible pour la capture des gouttes. Par exemple, le vulpin des champs, qui présente une petite surface foliaire (limitation du LAI) enroulée et un port dressé à de jeunes stades de croissance (2-3 feuilles), figure parmi les adventices problématiques en céréaliculture. En outre, les impacts des gouttes sont modifiés car cette inclinaison du feuillage induit une composante de vitesse tangentielle à l'impact et, par conséquent, une réduction de la composante normale prépondérante lors de l'impact de la goutte (Massinon et al., 2012a). Lors d'études en laboratoire sur vulpin, il a été montré que la proportion du rebond augmente lors de l'inclinaison de la surface foliaire de l'horizontal à la verticale pour une bouillie de tension de surface faible (cas de l'eau). Dans le cas d'une bouillie contenant un surfactant non ionique, une augmentation de la proportion de la fragmentation totale a été observée (Massinon et al., 2012a).

Avant de procéder au traitement envisagé, la technique d'application et la formulation de la bouillie doivent être adaptées en fonction des caractéristiques de la surface à traiter. Par ailleurs, la rétention des produits pulvérisés sur les surfaces super-hydrophobes est également influencée par les caractéristiques des gouttes (diamètre, vitesse et direction des gouttes). Le paragraphe suivant discutera l'effet des caractéristiques du spray sur la rétention.

\subsection{Effet des caractéristiques du jet sur les dépôts}

Impact de la taille et de la vitesse des gouttes (en fonction de la formulation de la bouillie) sur la rétention. De nombreuses études de laboratoires réalisées à l'échelle microscopique avec une buse hydraulique (Teejet XR 11003) ont mis en évidence l'effet de la taille des gouttes et de la formulation de bouillies sur la rétention en particulier sur des surfaces super-hydrophobes. Des histogrammes présentant le type d'impact en fonction de la vitesse des gouttes et 
de leurs diamètres ainsi que la nature de la surface ont été établis (Massinon et al., 2013 ; Boukhalfa et al., 2014). Pour une formulation à faible tension de surface, plus le diamètre et la vitesse de la goutte augmentent (de $3 \mathrm{~m} \cdot \mathrm{s}^{-1}$ jusque $10 \mathrm{~m} \cdot \mathrm{s}^{-1}$ avant l'impact), plus son énergie cinétique augmente et l'impact de la goutte subit une transition de l'adhésion au rebond (gouttes de diamètres < à $300 \mu \mathrm{m}$ ) puis à la fragmentation (gouttes de diamètres $>300 \mu \mathrm{m}$ ). La baisse de la tension de surface du liquide améliore les dépôts sur les cibles super-hydrophobes, en évitant le rebond de gouttes de taille moyenne entre $200 \mu \mathrm{m}$ et $350 \mu \mathrm{m}$ (Massinon et al., 2012b ; Massinon et al., 2013 ; Boukhalfa et al., 2014). Néanmoins, ces pratiques peuvent entrainer le ruissellement des gouttes sur des surfaces moins hydrophobes. En effet, l'étalement de la goutte aboutit à la création d'un film continu qui finit par tomber de la feuille. En outre, les gouttes dépassant les $350 \mu \mathrm{m}$, ayant une énergie cinétique élevée par rapport à leur tension de surface (correspondant à un nombre de Weber $\mathrm{We}^{1}$ élevé), ont une tendance à se fragmenter sur des surfaces super-hydrophobes malgré l'ajout d'un tensio-actif à la bouillie (Massinon et al., 2013 ; Boukhalfa et al., 2014). L'utilisation de grosses gouttes entraine une réduction du nombre d'impacts par unité de surface et nuit par conséquent au processus de rétention dans les premiers stades de croissance de la cible, et ce d'autant plus que la canopée est peu dense. Par ailleurs, les gouttes satellites qui se forment à partir de la fragmentation de la grosse goutte peuvent être interceptées de nouveau par le feuillage au voisinage dans le cas des LAI élevés (Butler Ellis et al., 2004).

De l'autre coté du spectre des gouttes, les petites gouttes de taille inférieure à $100 \mu \mathrm{m}$ constituent une bonne alternative pour augmenter la couverture d'une part et pour obtenir une distribution homogène de ces gouttes sur cette surface d'autre part (Knoche, 1994 ; Cox et al., 2000 ; Massinon et al., 2013). Face aux exigences actuelles des législations relatives à l'emploi des produits phytosanitaires et à la protection de l'environnement, une distribution mono-disperse de tailles de gouttes peut être la bonne alternative pour atteindre ces objectifs.

En sus de la taille des gouttes, la trajectoire de la goutte demeure une composante primordiale dans le processus de la rétention.

Impact de la trajectoire des gouttes sur la rétention. La trajectoire de la goutte, avec la variabilité de l'orientation foliaire, affecte les angles d'impacts des gouttes sur le feuillage. Certains modèles simulant la capture des gouttes émises par des buses hydrauliques ont été établis et permettent de simuler l'impact de la trajectoire de la goutte sur la surface cible en fonction des conditions externes (évaporation et turbulence) (Cox et al., 2000; Forster et al., 2005; Massinon et al., 2014 ; Massinon et al., 2015). Comme expliqué au § 2.1., l'orientation verticale de la cible atténue la composante normale de la vitesse des gouttes émises verticalement au-dessus de la cible. Dans le cadre du désherbage chimique du vulpin, l'inclinaison du jet de $60^{\circ}$ vers l'avant par rapport à la verticale (buse hydraulique) induit une augmentation de la rétention des herbicides appliqués (Butler Ellis et al., 2007 ; Jensen, 2007 ; Jensen, 2012). En outre, des buses à double sortie, produisant deux jets plats avec un angle de $60^{\circ}$ entre les deux projections vers l'avant et l'arrière, ont été développées. L'usage de ces buses vise à avoir une bonne couverture des dépôts sur ce genre de cibles. En effet, cette buse pourrait être un dispositif intermédiaire en terme d'orientation du jet. Cependant, cette pratique peut susciter des problèmes de dérive car l'orientation verticale des jets est un point clé pour l'atténuation de la dérive. Peu de documentation sur la potentialité de ces buses en matière de rétention et de dérive est présente dans la littérature.

D'autre part, la CDA est reconnue pour un meilleur contrôle de la taille et de la trajectoire des gouttes émises grâce à la maitrise de la formation des ligaments (nombre et diamètre) et à une vitesse d'éjection homogène des gouttes, comparée aux buses hydrauliques (Frost, 1981; Matthews, 1992). Cependant, les jets ont été émis horizontalement par rapport au sol et, par conséquent, les gouttes sédimentent sous l'effet de la pesanteur et suite à l'atténuation de leur vitesse d'éjection due aux frottements dans l'air (Matthews, 1992). L'inclinaison du jet du CDA, avec de bons réglages en termes de taille, vitesse et direction d'éjection des gouttes, pourrait être une voie potentielle pour l'amélioration des dépôts, surtout que les jets inclinés ont été prouvés bénéfiques pour la rétention. Par ailleurs, jusqu'à présent, peu de recherches ont traité l'impact de l'inclinaison des jets du CDA différente de l'horizontale sur la retention.

La réduction du spectre de tailles des gouttes peut induire une minimisation de la proportion de fines gouttes sensibles à la dérive et une augmentation de la proportion des gouttes de tailles optimales pour la rétention. De nouveaux réglages de l'atomiseur rotatif en termes du choix du $\mathrm{DV}_{50}$ et d'orientation du jet en fonction de la formulation de la bouillie pourraient satisfaire les objectifs actuels de la pulvérisation de précision.

Dans la suite, l'effet de la réduction des volumes à l'hectare sur la rétention à l'aide de la CDA, comparé aux buses hydrauliques, est étudié.

\footnotetext{
${ }^{1} \mathrm{We}=\frac{p v D}{\sigma}, p:$ densité du fluide, $v:$ vitesse de la goutte $\left(\mathrm{m} \cdot \mathrm{s}^{-1}\right), D:$ diamètre initial de la goutte $(\mathrm{m}), \sigma:$ tension de surface du fluide $\left(\mathrm{N} \cdot \mathrm{m}^{-1}\right)$.
} 


\subsection{Impact de la réduction des volumes à l'hectare sur l'efficience des traitements}

L'application des produits phytosanitaires de contact, en fonction de la vigueur de la canopée de la plante, exige des volumes à l'hectare élevés jusque $4001 \cdot$ ha $^{-1}$ (Taylor, 1981 ; Knoche, 1994), en vue de maintenir une fréquence d'impact des gouttes optimale pour leurs couvertures sur la cible. Cependant, selon des tests empiriques, des réductions de volume de $2501 \cdot \mathrm{ha}^{-1}$ jusque $1251 \cdot h^{-1}$ peuvent être effectuées sans affecter la performance des dépôts (Skuterud et al., 1988 ; Butler Ellis et al., 2007). En dessous de ces volumes $\left(<1001 \cdot h^{-1}\right)$, cette performance est détériorée car cette pratique engendre une augmentation de la variabilité de dépôts et la probabilité de pertes hors de la cible (Knoche, 1994 ; Butler Ellis et al., 2007). En outre, les interactions existantes entre le volume appliqué et la taille des gouttes influençant la performance de ces produits ont été établies. En effet, la variabilité des dépôts liée à la taille des gouttes est importante dans le cadre des applications à bas volumes. De même, la variabilité liée aux volumes appliqués est importante avec des gouttes de grosses tailles et inversement (Butler Ellis et al., 2007).

Quant à la technique d'application, la comparaison de la réduction de volumes entre la CDA et l'application hydraulique est difficile car la distance d'écartement des buses $(50 \mathrm{~cm}$ pour les buses hydrauliques et $1 \mathrm{~m}$ pour la CDA) se traduit par des différences au niveau des volumes appliqués ( Skuterud et al., 1988 ; Knoche, 1994 ; Holland et al., 1997 ; Butler Ellis et al., 2007). Ces comparaisons pour des volumes différents ont abouti à des résultats contradictoires, comme le montre le tableau 1. Butler Ellis et al. (2007) ont investigué cette dualité pour le même volume à l'hectare sur des cibles synthétiques verticales et les résultats ont montré que la différence entre les techniques d'applications en termes de moyenne de dépôts est minime lors de la réduction des volumes de $2001 \cdot \mathrm{ha}^{-1}$ à $501 \cdot \mathrm{ha}^{-1}$. Pour les applications à des volumes de $251 \cdot \mathrm{ha}^{-1}$, la CDA entraine des dépôts plus importants par rapport aux buses hydrauliques et anti-dérives (induction d'air). Ceci est probablement dû à la trajectoire des gouttes qui possèdent une composante horizontale favorisant leur impact sur ce type de cible à port dressé (Tableau 2) (Butler Ellis et al., 2007).

Plusieurs études ont été bâties sur l'hypothèse que les petites cibles peuvent recevoir des doses inadéquates lors des pulvérisations à des bas volumes. Cette dernière ne peut pas être seulement validée par la moyenne des dépôts mais aussi au moyen du coefficient de variation $(\mathrm{CV})$ et de la couverture de ces dépôts (Tableau 3 ) (Butler Ellis et al., 2007). À titre d'exemple, le CV augmente avec la réduction des volumes, surtout pour des cibles verticales synthétiques. En termes de techniques d'application, les buses à induction d'air résultent en une variabilité plus importante que la CDA et que les buses à jets plats pour des cibles synthétiques horizontales et verticales. Lorsque le raygrass au stade 2-3 feuilles a été investigué, la même tendance a été observée pour les différentes techniques d'applications, sauf dans le cas de la CDA où le CV a diminué lors de la réduction de volume de 50 l.ha ${ }^{-1}$ à $251 \cdot$ ha $^{-1}$ (Butler Ellis et al., 2007). Par ailleurs, la couverture des dépôts augmente avec l'augmentation des volumes appliqués, malgré les fines gouttes présentes dans le jet à des bas volumes pour la CDA et les buses hydrauliques. Les buses à induction d'air assurent un niveau de couverture acceptable car les gouttes laissent de longues traces sur la cible lors de l'impact. Les buses hydrauliques présentent toujours une meilleure couverture pour tous les volumes testés (Tableau 3) (Butler Ellis et al., 2007).

Il est incontestable que la comparaison de ces deux techniques d'applications est difficile à cause essentiellement des réglages des atomiseurs de ces deux techniques, car les tests sont généralement

Tableau 1. Comparaison de différentes techniques d'application en termes de rétention - Comparison of different spray applications in termes of spray retention.

\begin{tabular}{|c|c|c|c|c|c|}
\hline $\begin{array}{l}\text { Technique } \\
\text { d'application }\end{array}$ & Atomiseur & $\begin{array}{l}\text { Informations } \\
\text { supplémentaires }\end{array}$ & $\begin{array}{l}\text { Volume } \\
\text { appliqué }\left(1 \cdot h^{-1}\right)\end{array}$ & $\begin{array}{l}\mathbf{D V}_{\mathbf{5 0}} \\
(\mu \mathrm{m})\end{array}$ & $\begin{array}{l}\text { Niveau de contrôle } \\
\text { des adventices } \\
(\text { contrôle }=100)\end{array}$ \\
\hline $\mathrm{CDA}^{1}$ & Micromax (Micron crop) & $\begin{array}{l}2000 \text { tours } \cdot \mathrm{min}^{-1} \\
2000 \text { tours } \cdot \mathrm{min}^{-1}\end{array}$ & $\begin{array}{l}28 \\
56\end{array}$ & $\begin{array}{l}231 \\
293\end{array}$ & $\begin{array}{l}60 \\
90\end{array}$ \\
\hline Conventionnelle $^{1}$ & Flat 65015 (Spraying systems) & & 187 & 241 & 30 \\
\hline $\mathrm{CDA}^{2}$ & Micromax Mk III (Micron sprayer) & $\begin{array}{l}3500 \text { tours } \cdot \mathrm{min}^{-1} \\
2000 \text { tours } \cdot \mathrm{min}^{-1} \\
2000 \text { tours } \cdot \mathrm{min}^{-1} \\
2000 \text { tours } \cdot \mathrm{min}^{-1}\end{array}$ & $\begin{array}{l}25 \\
25 \\
30 \\
40\end{array}$ & $\begin{array}{l}105 \\
158 \\
233\end{array}$ & $\begin{array}{l}50 \\
35 \\
47 \\
44\end{array}$ \\
\hline Conventionnelle $^{2}$ & Teejet 11004 & & 250 & 355 & 78 \\
\hline
\end{tabular}

${ }^{1}$ : Gebhardt et al., $1985 ;{ }^{2}$ : Skuterud et al., 1988. 
Tableau 2. Impact de la réduction du volume appliqué sur la rétention - Impact of the reduction of the volume applied on spray retention (Butler Ellis et al., 2007).

\begin{tabular}{|c|c|c|c|c|c|}
\hline $\begin{array}{l}\text { Volume appliqué } \\
\left(1 \cdot \text { ha }^{-1}\right)\end{array}$ & Type d'application & $\begin{array}{l}\text { Atomiseur/vitesse de } \\
\text { rotation du disque }\end{array}$ & Pression & $\begin{array}{l}\text { Hauteur de } \\
\text { l'atomiseur } \\
(\mathrm{mm})\end{array}$ & $\begin{array}{l}\text { Rétention par } \\
\text { cible ( } \mu 1 \text { pour } \\
1001 \cdot \text { ha }^{-1} \text { appliqué) }\end{array}$ \\
\hline 25 & Conventionnelle & FF 11001 & 1,80 & 50 & 1,18 \\
\hline 34 & Induction d'air & Bubblejet 015 & 1,80 & 50 & 0,90 \\
\hline 36 & Induction d'air & Drift Beta 015 & 1,80 & 50 & 0,92 \\
\hline 25 & $\mathrm{CDA}$ & Micromax, 5000 tours $\cdot \mathrm{min}^{-1}$ & 1,25 & 50 & 2,50 \\
\hline 50 & $\begin{array}{l}\text { Conventionnelle } \\
\text { Induction d'air } \\
\text { Induction d'air } \\
\text { CDA }\end{array}$ & $\begin{array}{l}\text { FF } 11002 \\
\text { Bubblejet } 015 \\
\text { Injet } 02 \\
\text { Micromax, } 3500 \text { tours } \cdot \mathrm{min}^{-1}\end{array}$ & $\begin{array}{l}1,80 \\
3,80 \\
1,80 \\
2,55\end{array}$ & $\begin{array}{l}50 \\
50 \\
50 \\
50\end{array}$ & $\begin{array}{l}0,87 \\
0,67 \\
1,02 \\
0,92\end{array}$ \\
\hline 100 & $\begin{array}{l}\text { Conventionnelle } \\
\text { Induction d'air } \\
\text { Induction d'air } \\
\text { CDA }\end{array}$ & $\begin{array}{l}\text { FF } 11002 \\
\text { Bubblejet } 015 \\
\text { Injet } 02 \\
\text { Micromax, } 3500 \text { tours } \cdot \mathrm{min}^{-1}\end{array}$ & $\begin{array}{l}2,00 \\
4,00 \\
2,00 \\
2,55\end{array}$ & $\begin{array}{l}50 \\
50 \\
50 \\
50\end{array}$ & $\begin{array}{l}0,98 \\
0,79 \\
0,82 \\
0,83\end{array}$ \\
\hline 200 & $\begin{array}{l}\text { Conventionnelle } \\
\text { Induction d'air } \\
\text { Induction d'air } \\
\text { CDA }\end{array}$ & $\begin{array}{l}\text { FF } 11004 \\
\text { Bubblejet } 03 \\
\text { Drift Beta } 04 \\
\text { Micromax, } 2000 \text { tours } \cdot \mathrm{min}^{-1}\end{array}$ & $\begin{array}{l}2,00 \\
3,50 \\
2,00 \\
1,40\end{array}$ & $\begin{array}{l}50 \\
50 \\
50 \\
50\end{array}$ & $\begin{array}{l}0,92 \\
0,94 \\
1,38 \\
1,01\end{array}$ \\
\hline
\end{tabular}

Tableau 3. Coefficients de variations et couvertures des jets issus de différentes techniques d'applications. Les réglages des atomiseurs testés sont les mêmes que ceux indiqués au tableau 1 - Coefficients of variations and coverages of sprays emitted from different application techniques. Settings of tested atomisers are the same as those indicated in table 1 (Butler Elis et al., 2007).

\begin{tabular}{|c|c|c|c|c|}
\hline & Volume appliqué $\left(1 \cdot \mathrm{ha}^{-1}\right)$ & Type d'application & Cible horizontale & cible verticale \\
\hline \multirow[t]{5}{*}{$\begin{array}{l}\text { Coefficient de variation des dépôts } \\
\text { normalisé à } 1001 \cdot \mathrm{ha}^{-1}(\mathrm{CV})(\%)\end{array}$} & 25 & $\begin{array}{l}\text { Conventionnelle } \\
\text { CDA }\end{array}$ & $\begin{array}{l}21,30 \\
24,90\end{array}$ & $\begin{array}{l}28,40 \\
37,60\end{array}$ \\
\hline & 36 & Induction d'air & 37,00 & 56,90 \\
\hline & 50 & $\begin{array}{l}\text { Conventionnelle } \\
\text { Induction d'air } \\
\text { CDA }\end{array}$ & $\begin{array}{l}18,60 \\
45,50 \\
38,80\end{array}$ & $\begin{array}{l}20,60 \\
91,00 \\
33,40\end{array}$ \\
\hline & 100 & $\begin{array}{l}\text { Conventionnelle } \\
\text { Induction d'air } \\
\text { CDA }\end{array}$ & $\begin{array}{l}17,00 \\
37,00 \\
21,90\end{array}$ & $\begin{array}{l}18,80 \\
70,50 \\
29,20\end{array}$ \\
\hline & 200 & $\begin{array}{l}\text { Conventionnelle } \\
\text { Induction d'air } \\
\text { CDA }\end{array}$ & $\begin{array}{l}20,30 \\
22,90 \\
19,40\end{array}$ & $\begin{array}{l}18,90 \\
55,80 \\
24,70\end{array}$ \\
\hline \multirow[t]{5}{*}{ Couverture (\%) } & 25 & $\begin{array}{l}\text { Conventionnelle } \\
\text { CDA }\end{array}$ & 14,55 & $\begin{array}{l}1,83 \\
1,75\end{array}$ \\
\hline & 36 & Induction d'air & 8,27 & 4,33 \\
\hline & 50 & $\begin{array}{l}\text { Conventionnelle } \\
\text { Induction d'air } \\
\text { CDA }\end{array}$ & $\begin{array}{l}26,49 \\
13,09\end{array}$ & $\begin{array}{l}4,50 \\
4,50 \\
2,92\end{array}$ \\
\hline & 100 & $\begin{array}{l}\text { Conventionnelle } \\
\text { Induction d'air } \\
\text { CDA }\end{array}$ & $\begin{array}{l}47,23 \\
31,94\end{array}$ & $\begin{array}{l}9,08 \\
6,83 \\
4,75\end{array}$ \\
\hline & 200 & $\begin{array}{l}\text { Conventionnelle } \\
\text { Induction d'air } \\
\text { CDA }\end{array}$ & $\begin{array}{l}68,38 \\
50,79\end{array}$ & $\begin{array}{l}19,58 \\
15,92 \\
13,83\end{array}$ \\
\hline
\end{tabular}


effectués à des volumes à l'hectare et des tailles de gouttes différentes. En outre, l'architecture de la cible en elle-même représente une source de variabilité, puisque les petites gouttes de la CDA n'arrivent pas à pénétrer la canopée dense des céréales (Skuterud et al., 1988). Néanmoins, les recommandations de l'augmentation des volumes appliquées en vue d'améliorer la pénétration de ces gouttes dans les canopées denses ne sont pas validées (Butler Ellis et al., 2014).

Toutefois, l'efficacité des produits phytosanitaires, malgré la grande proportion des gouttes qui arrivent à adhérer à la surface cible, reste limitée par les pertes hors de la cible connues essentiellement par le phénomène de dérive. Dans la suite, les potentialités de dérive des jets agricoles issues de la CDA et de la technique hydraulique sont investiguées.

\section{ASPECT DÉRIVE}

La dérive correspond au phénomène de transport des gouttes ou des vapeurs hors de la zone ciblée par le traitement. Ce transport est provoqué par les mouvements d'air (BCPC, 1986 ; Al Heidary et al., 2014). Les principaux paramètres influençant la dérive sont les caractéristiques des gouttes (taille, vitesse et direction d'éjection) (Solie et al., 1986 ; Qi et al., 2008), les conditions météorologiques externes (vitesse du vent) (Gil et al., 2005) et la hauteur de la buse par rapport au sol (Baetens et al., 2009). Dans cette partie, l'accent est seulement mis sur l'interaction des caractéristiques des gouttes avec le vent en fonction de la technique d'application.

\subsection{Paramètres impliqués et mesures de mitigation de dérive}

La dérive est fortement liée aux paramètres décrivant la distribution des tailles des gouttes, notamment le $\mathrm{DV}_{50}$ (Matthews, 1992; Qi et al., 2008) et la proportion en volume des gouttes de taille inférieure à $100 \mu \mathrm{m}$ (V100) (Matthews, 1992 ; Van De Zande et al., 2002) ou $200 \mu \mathrm{m}$ (Hewitt et al., 1998) en fonction de la vitesse du vent. En effet, pour des buses hydrauliques, le potentiel de dérive, mesuré à $2 \mathrm{~m}$ de la buse et à une vitesse du vent de $2 \mathrm{~m} \cdot \mathrm{s}^{-1}$ et exprimé en pourcentage du volume appliqué, diminue exponentiellement lorsque le $\mathrm{DV}_{50}$ augmente (Al Heidary et al., 2014). Quant à la CDA, des études dans un tunnel de dérive ont montré que le potentiel de dérive, dans les mêmes conditions précédentes, augmente alors que le $\mathrm{DV}_{50}$ diminue de 244 à $140 \mu \mathrm{m}$ (Qi et al., 2008). Cette augmentation de la dérive est de plus en plus forte à mesure que la vitesse du vent augmente jusque $5 \mathrm{~m} \cdot \mathrm{s}^{-1}$. Les jets, ayant un $\mathrm{DV}_{50}$ en dessous de $300 \mu \mathrm{m}$, présentent un pourcentage élevé de gouttes sensibles à la dérive $(<100 \mu \mathrm{m})$. Par ailleurs, pour un même $\mathrm{DV}_{50}$ de $207 \mu \mathrm{m}$, la CDA possède une proportion de gouttes $<100 \mu \mathrm{m}$ faible par rapport à la buse hydraulique. Les gouttes de la CDA dérivent moins à $2 \mathrm{~m}$ de la buse pour une vitesse de vent à $2 \mathrm{~m} \cdot \mathrm{s}^{-1}$ par rapport à la buse hydraulique. Néanmoins, ces gouttes seront aéroportées plus que celles de la buse hydraulique lorsque la vitesse du vent augmente de $2 \mathrm{~m} \cdot \mathrm{s}^{-1}$ jusque $5 \mathrm{~m} \cdot \mathrm{s}^{-1}$ (Tableau 4).

Ensuite, le deuxième paramètre affectant la dérive est la vitesse de la goutte. En effet, la goutte atteint sa vitesse maximale à son point d'émission puis elle ralentit lors de son transport vers la cible, du fait des frottements avec l'air. A titre d'exemple, pour les buses hydrauliques, les gouttes ayant des $\mathrm{DV}_{50}$ inférieurs à 300 microns possèdent une vitesse moyenne (médiane) entre 2 et $4 \mathrm{~m} \cdot \mathrm{s}^{-1}$ (buses à jets plats), tandis que ceux de $\mathrm{DV}_{50}$ supérieurs possèdent une vitesse moyenne (médiane) entre 4 et $7 \mathrm{~m} \cdot \mathrm{s}^{-1}$ (buses antidérives et à injection d'air) (Nuyttens et al., 2009 ; Al Heidary et al., 2014) mesurée à $50 \mathrm{~cm}$ de distance. Ces vitesses sont petites comparées à celles de la CDA qui dépendent de la vitesse de rotation et du débit. Les vitesses élevées permettent aux gouttes de réduire leur temps de parcours et par conséquent de limiter l'impact des mouvements d'air sur leur trajectoire (Matthews, 1992 ; Qi et al.,

Tableau 4. Effet de $\mathrm{V}_{10}$, de $\mathrm{V}_{50}(\mu \mathrm{m})$, du span et de la vitesse du vent sur la dérive - Effect of $V_{10}$, $V_{50}(\mu \mathrm{m})$, span and wind velocity on spray drift (Qi et al,. 2008).

\begin{tabular}{|c|c|c|c|c|c|c|c|c|c|}
\hline & \multirow[t]{2}{*}{ Atomiseur } & \multirow{2}{*}{$\begin{array}{l}\text { Pression } \\
\text { (bar) }\end{array}$} & \multirow{2}{*}{$\begin{array}{l}\mathbf{D V}_{10} \\
(\mu \mathrm{m})\end{array}$} & \multirow{2}{*}{$\begin{array}{l}\mathbf{D V}_{\mathbf{5 0}} \\
(\mu \mathrm{m})\end{array}$} & \multirow[t]{2}{*}{ Span } & \multicolumn{4}{|c|}{ Estimation de la dérive } \\
\hline & & & & & & $\%, 2 \mathrm{~m} \cdot \mathrm{s}^{-1}$ & $\%, 3 \mathrm{~m} \cdot \mathrm{s}^{-1}$ & $\%, 4 \mathrm{~m} \cdot \mathrm{s}^{-1}$ & $\%, 5 \mathrm{~m} \cdot \mathrm{s}^{-1}$ \\
\hline \multirow{4}{*}{$\begin{array}{l}\text { Buses } \\
\text { hydrauliques }\end{array}$} & FF110/0,49/4,5 & 4,5 & 101,85 & 164 & 0,82 & 22,50 & 33,50 & 42,67 & 48,00 \\
\hline & FF110/0,86/3,5 & 3,5 & 130,27 & 207 & 0,86 & 10,50 & 18,83 & 24,17 & 26,17 \\
\hline & FF110/1,46/2,5 & 2,5 & 152,98 & 255 & 0,93 & 4,00 & 8,17 & 11,33 & 14,00 \\
\hline & FF110/1,96/2,0 & 2,0 & 171,00 & 302 & 0,95 & 2,50 & 5,50 & 7,67 & 9,33 \\
\hline \multirow{3}{*}{$\mathrm{CDA}$} & 2000 tours $\cdot \mathrm{min}^{-1}$ & 0,6 & 180,13 & 244 & 0,53 & 2,17 & 5,33 & 18,50 & 39,33 \\
\hline & 3500 tours $\cdot \mathrm{min}^{-1}$ & 0,6 & 148,36 & 207 & 0,54 & 6,50 & 31,17 & 62,00 & 84,67 \\
\hline & 5000 tours $\cdot \mathrm{min}^{-1}$ & 1,2 & 91,15 & 140 & 0,69 & 33,50 & 84,00 & 94,67 & 97,83 \\
\hline
\end{tabular}


2008). Néanmoins, la direction d'éjection des gouttes affecte aussi cette trajectoire. En effet, les jets issus des atomiseurs rotatifs sont émis horizontalement, induisant une faible composante verticale de la vitesse de la goutte par rapport aux buses hydrauliques. Les profils verticaux de dérive à $2 \mathrm{~m} \cdot \mathrm{s}^{-1}$ montrent que les gouttes émises horizontalement dérivent de manière significativement plus importante que lorsque les gouttes sont émises verticalement. Aussi, ces gouttes demeurent plus exposées aux forces de frottement induites par l'air malgré leurs vitesses d'éjection élevées (Dombrowski et al., 1973 ; Bode et al., 1983 ; Gebhardt, 1988 ; Qi et al., 2008).

Des mesures de protection des jets issus de la CDA inscrites dans le cadre des stratégies de mitigation de dérive ont été investiguées. La première tentative a été bâtie sur la conception de boucliers dont l'angle d'ouverture varie entre $120^{\circ}$ et $140^{\circ}$ (Gebhardt, 1988). Un modèle mathématique a été développé afin de prédire la distribution du jet émis horizontalement à partir d'un disque rotatif de surface lisse (Goering et al., 1972). Néanmoins, cette pratique a créé des vortex lors du déplacement de l'atomiseur induisant ainsi une turbulence dans l'air et des problèmes de fragmentation du jet aux angles d'ouvertures du bouclier (Matthews, 1992). En termes de buses hydrauliques, l'effet des boucliers montés sur des rampes de pulvérisation sur la dérive dans un tunnel de dérive a été investigué. Le potentiel de dérive a été évalué par le calcul du centre de gravité des dépôts DC (une petite valeur de DC indique qu'une grande partie du jet est déposée près de la buse). Les résultats ont montré que les boucliers testés ont réduit la valeur de DC pour toutes les buses testées. Les modèles les moins efficaces ont contribué à une réduction de $13 \%$ de la valeur de DC, tandis que les boucliers à double face ont été les plus efficaces avec une réduction de $59 \%$ du DC comparés à des buses sans boucliers testés dans les mêmes conditions (Ozkan et al., 1997).

La deuxième tentative a été fondée sur l'hypothèse d'augmenter la composante verticale de la vitesse des gouttes en vue de réduire les effets du vent de direction horizontale. Un modèle français (Girojet, Tecnoma), présentant un bouclier dont l'angle d'ouverture est de $140^{\circ}$ et de direction verticale, a été conçu. Un modèle mathématique a été développé en vue de prédire la distribution du jet émis verticalement et il a été validé par des mesures de terrain (Solie et al., 1986). Par ailleurs, cette technologie n'a pas été documentée de manière importante en termes de potentiel de dérive. Quelques publications ont cité sa faible sensibilité à la dérive comparée aux disques horizontaux ou aux buses de pressions (Bouchet, 1982; Morel, 1982). D'autres mesures, notamment sur l'abaissement de la hauteur de la rampe, peuvent être envisagées.

\subsection{Classification des risques de dérive des jets agricoles}

La classification de la potentialité de dérive des jets agricoles est la base des stratégies optant pour la réduction de dérive de produits phytosanitaires. La taille des gouttes est considérée comme le critère principal pour l'établissement de cette classification. Le potentiel de dérive des buses à jets plats est divisé en quatre classes (très élevé, élevé, moyen, faible) à l'aide de trois buses de références (FF110/0.49/4.5 ; FF110/1.2/3.0 ; FF110/1.96/2.0) utilisées comme limites de ces quatre classes (Hoffmann et al., 2005). Qi et al. (2008) ont utilisé cette classification en gardant les mêmes buses de références en vue d'établir une classification du potentiel de dérive des jets issus des atomiseurs rotatifs sans boucliers et de directions horizontales dans un tunnel de dérive. Ces auteurs ont développé les mêmes classes citées précédemment, en fonction de la vitesse de rotation du disque $\left(2000,3500\right.$ et 5000 tours. $\left.\mathrm{min}^{-1}\right)$ et de la vitesse du vent (mêmes sens et direction que les jets allant de 2 à $5 \mathrm{~m} \cdot \mathrm{s}^{-1}$ ). Les profils de dérive verticale à $2 \mathrm{~m}$ de la buse montrent que le spray de la CDA produit plus de dérive que ceux des buses à jets plats, sauf les sprays des disques rotatifs (CDA), réglés à 2000 tours. $\mathrm{min}^{-1}$ (émettant de grosses gouttes) avec un débit de $0,481 \cdot \mathrm{min}^{-1}$ et soumis à une vitesse de vent inférieure à $2 \mathrm{~m} \cdot \mathrm{s}^{-1}$. En dehors de ces conditions, la sensibilité des jets horizontaux aux courants d'air dépasse celle des autres buses hydrauliques montées verticalement.

\section{DISCUSSION}

La CDA présente un avantage double de réduction de l'étendue du spectre et du volume appliqué. De nombreuses recherches menées pour améliorer la qualité du jet vers un span réduit et le contrôle de la trajectoire de la goutte, ont abouti à la conception de dents usinées directement au bord du disque. Cependant, ces dernières se sont avérées sensibles à l'usure et, par conséquent, elles modifient drastiquement les propriétés du jet. Aussi, la conception des atomiseurs rotatifs munis de boucliers dans le but d'émettre un jet similaire à celui des buses hydrauliques, a induit une modification de la qualité du jet émis dû aux éclaboussures produites au niveau de deux fentes de boucliers. Peu de recherches ont investigué l'efficacité de ce type d'atomiseur en termes de rétention et de dérive.

En sus de ces défaillances de conception, du prix élevé et de la maintenance de cette technologie, il s'est avéré que la réticence au niveau de la céréaliculture visà-vis de la CDA est due essentiellement à des réglages erronés de ces atomiseurs en termes de taille des gouttes et de directions d'éjections. En effet, les jets issus de ces atomiseurs sont émis parallèlement ou avec une 
légère inclinaison par rapport au sol et caractérisés par un $\mathrm{DV}_{50}$ inapproprié pour la maximisation des dépôts et la limitation de la dérive. Par ailleurs, les recherches à l'échelle microscopique reliant la taille de la goutte et les propriétés de la bouillie ont montré que la taille optimale de la goutte pour la rétention sur une cible d'orge (limite entre l'adhésion et la fragmentation) est de $300 \mu \mathrm{m}$, en fonction de son énergie d'impact, de la dose de tensio-actif et de la pression appliquée (Boukhalfa et al., 2014). Ce type de $\mathrm{DV}_{50}$ induit pour la distribution granulométrique de la CDA une réduction de la proportion en volume de gouttes sujettes à la dérive.

Aussi, le choix approprié du $\mathrm{DV}_{50}$ peut être combiné avec l'angle d'inclinaison du jet. En effet, les jets, issus des buses hydrauliques et inclinés de $60^{\circ}$ vers l'avant par rapport à la verticale, se sont avérés efficaces sur des cibles à port dressé. Or, cette pratique suscite des problèmes de dérive. Dès lors, un jet de $\mathrm{DV}_{50}$ autour de $300 \mu \mathrm{m}$, de faible proportion de gouttes sensibles à la dérive et émis $60^{\circ}$ vers l'avant par rapport à la verticale, peut être une alternative clé pour limiter la dérive et maximiser les dépôts.

En outre, l'application d'une distribution monodisperse est une voie prometteuse pour limiter les pertes hors cibles. Sur cette base, des études récentes ont abouti au contrôle dimensionnel de la taille de gouttes par l'application d'ondes acoustiques. Cette technique a été fondée sur l'application d'une vibration mécanique ou acoustique dans des conditions bien définies (amplitude et fréquence des ondes) sur un écoulement au bord d'un disque rotatif de petite dimension $(1 \mathrm{~cm}$ de diamètre). Cette vibration se superpose à l'écoulement et aboutit à une rupture forcée des ligaments et par la suite à des gouttes de tailles homogènes (Chicheportiche et al., 2004 ; Zainoun et al., 2004). Cependant, cette pratique reste limitée et n'a pas pu être extrapolée au niveau de la pulvérisation agricole à cause de sa limitation à de faibles débits.

\section{CONCLUSIONS}

La CDA demeure un outil efficace de contrôle de span et de la trajectoire des gouttes. L'échec commercial de cette technologie est dû essentiellement aux mauvais réglages qui datent des années 1980 et 1990. De nouveaux réglages en termes de choix du $\mathrm{DV}_{50}$ du jet, des caractéristiques physicochimiques de la bouillie et de la direction du jet sont une bonne alternative pour la relance de la CDA et pour palier aux défaillances des buses à jets plats et anti-dérive en termes de rétention et de dérive.

Cette technologie présente la base sur laquelle de nouvelles pistes peuvent être bâties en vue de réaliser les défis de l'agriculture de précision à court terme. Ces pistes doivent investiguer des solutions pratiques pour l'usure des dents à la sortie du disque par le biais de sorties plus souples d'une part, et étudier l'organisation de la rupture des ligaments liquides en vue d'avoir une distribution granulométrique des gouttes plus étroite d'autre part.

\section{Remerciements}

Nous remercions l'Université de Liège - Gembloux Agro-Bio Tech, et plus particulièrement la plateforme d'AgricultureIsLife pour le financement de ce projet de recherche.

\section{Bibliographie}

Al Heidary M., Douzals J.P., Sinfort C. \& Vallet A., 2014. Influence of spray characteristics on potential spray drift of field crop sprayers: a literature review. Crop Prot., 63, 120-130.

Baetens K. et al., 2009. A validated 2-D diffusion-advection model for prediction of drift from ground boom sprayers. Atmos. Environ., 43, 1674-1682.

BCPC (British Crop Protection Council), 1986. Nozzle classification scheme. Alton, UK: BCPC.

Bédiaf H., Sabre R., Journaux L. \& Cointault F., 2015. Comparison of leaf surface roughness analysis methods by sensitivity to noise analysis. Biosyst. Eng., 136, 7786.

Bode L.E., Zimmerman T.L., Goering C.E. \& Gebhardt M.R., 1972. The effects of flow rate on the distribution pattern and drop-size spectrum of a spinning atomizer. Trans. ASAE, 15, 86-90.

Bode L.E., Butler B.J., Pearson S.L. \& Bouse L.F., 1983. Characteristics of the micromax rotary atomizer. Trans. ASAE, 26, 999-1005.

Bouchet C., 1982. Pulvérisation à volume réduit et bas volume en grandes cultures. Résultats de trois années d'experimentation. EPPO Bull., 13(3), 547-554.

Boukhalfa H.H., Massinon M., Belhamra M. \& Lebeau F., 2014. Contribution of spray droplet pinning fragmentation to canopy retention. Crop Prot., 56, 91-97.

Butler Ellis M.C., Webb D.A. \& Western N.M., 2004. The effect of different spray liquids on the foliar retention of agricultural sprays by wheat plants in a canopy. Pest Manage. Sci., 60, 786-794.

Butler Ellis M.C., Knight S. \& Miller P.C.H., 2007. Spray behaviour and efficacy of herbicides and fungicides applied to wheat at reduced volumes. HGCA project report 408. Kenilworth, UK: Agriculture and Horticulture Development Board Cereals \& Oilseeds.

Butler Ellis M.C., Lane A.G. \& O’Sullivan C.M., 2014. Distribution of spray applied to a cereal crop and the effect of application parameters on penetration. $H G C A$ project report 526. Kenilworth, UK: Agriculture and Horticulture Development Board Cereals \& Oilseeds. 
Chicheportiche J.M., Renaudeaux J.P. \& Zainoun N., 2004. Atomiseur à disque tournant d'aérosols calibrés. Demande brevet d'invention 0403679.

Clayton J., 1992. A new generation hand held spinning disc sprayer: the micron ulvat for small farmer crop protection. In: Proceedings of the IRCT cotton conference, February 1992, N'Djamena, Chad.

Clayton J.S., Bals T.E. \& Povey G.S., 1993. A new generation hand-held ulva sprayer. In: Proceedings of the $2^{\text {nd }}$ International symposium on pesticide application techniques, 22-24 September 1993, Strasbourg, France.

Cox S.J., Salt D.W., Lee B.E. \& Ford M.G., 2000. A model for the capture of aerially sprayed pesticide by barley. J. Wind Eng. Ind. Aerodyn., 87, 217-230.

Derksen R.C. \& Bode L.E., 1986. Droplet size comparisons from rotary atomisers. Trans. ASAE, 29, 1204-1207.

Doble S.J., Matthews G.A., Rutherford I. \& Southcombe E.S.E., 1985. A system for classifying hydraulic and other atomisers into categories of spray quality. In: Proceedings of the Brighton crop protection conference - weeds, 18-21 November, 1985, Brighton Metropole, England, 1125-1133. British Crop Protection Enterprises.

Dombrowski N. \& Lloyd T.L., 1973. Air drag on ligaments produced from rotating cups. J. Chem. Eng. Jpn., 6(4), 363-365.

Dombrowski N. \& Lloyd T.L., 1974. Atomisation of liquids by spinning cups. Chem. Eng. J., 8, 63-81.

Elbanna H., Rashed M.J.I. \& Ghazi M.A., 1984. Droplets from liquid sheets in an air stream. Trans. ASAE, 27, 677-679.

Forster W.A., Kimberley M.O. \& Zabkiewicz J.A., 2005. A universal spray droplet adhesion model. Trans. ASAE, 48(4), 1321-1330.

Frost A.R., 1981. Rotary atomization in the ligament formation mode. J. Agric. Eng. Res., 26, 63-78.

Gebhardt M.R., 1988. Rotary disk atomisation. Weed Technol., 2, 106-113.

Gil Y. \& Sinfort C., 2005. Emission of pesticides to the air during sprayer application: a bibliographic review. Atmos. Environ., 39, 5183-5193.

Goering C.E., Foster R.A., Bode L.E. \& Gebhardt M.R., 1972. Development of a shielded, spinning disk atomizer. Trans. ASAE, 15(5), 814-817.

Hewitt A.J., Valcore D.L., Teske M.E. \& Schick R.J., 1998. Drop size classifications for agricultural sprays. In: Proceedings of the $11^{\text {th }}$ Annual conference on liquid atomization and spray systems, May 1998, Sacramento, CA, USA.

Hoffmann W.C. \& Kirk I.W., 2005. Spray deposition and drift from two "medium" nozzles. Trans. ASAE, 48(1), 5-11.

Holland J.M., Jepson P.C., Jones E.C. \& Turner C., 1997. A comparison of spinning disc atomisers and flat fan pressure nozzles in terms of pesticide deposition and biological efficacy within cereal crops. Crop Prot., 16, 179-185.

Jensen P.K., 2007. Nonvertical spray angles optimize graminicide efficacy. Weed Technol., 21, 1029-1034.

Jensen P.K., 2012. Increasing efficacy of graminicides with a forward angled spray. Crop Prot., 32, 17-23.

Knoche M., 1994. Effect of droplet size and carrier volume on performance of foliage-applied herbicides. Crop Prot., 13, 163-178.

Massinon M., Boukhalfa H.H., Marechal P. \& Lebeau F., 2012a. The effect of leaf orientation on spray retention on blackgrass. In: Proceedings of the $11^{\text {th }}$ International conference on precision agriculture, 15-18 July 2012, Hyatt Regency, Indianapolis, USA.

Massinon M. \& Lebeau F., 2012b. Comparison of spray retention on synthetic superhydrophobic surface with retention on outdoor grown wheat leaves. In: Proceedings of the International advances in pesticide application: aspects of applied biology 114, 11 January 2012, Hof Wageningen, The Netherlands. Warwick, UK: Association of Applied Biologists, 261-268.

Massinon M.\&Lebeau F., 2013. Review of physicochemical processes involved in agrochemical spray retention. Biotechnol. Agron. Soc. Environ., 17, 494-504.

Massinon M. et al., 2014. Evaluation of process-driven spray retention model on early growth stage barley. In: Proceedings of the International conference of agricultural engineering, 6-10 July 2014, Zurich, Switzerland.

Massinon M. et al., 2015. Study of retention variability on an early growth stage herbaceous plant using a 3D virtual spraying model. Crop Prot., 78, 63-71.

Matthews G.A., 1992. Pesticide application methods. $2^{\text {nd }} \mathrm{ed.}$ Harlow, UK: Longman.

Morel M., 1982. Pulvérisation centrifuge à très bas volume : TG 412. EPPO Bull., 13(3), 363-364.

Nuyttens D. et al., 2009. Influence of nozzle type and size on drift potential by means of different wind tunnel evaluation methods. Biosyst. Eng., 103, 271-280.

Ozkan H.E. et al., 1997. Shields to reduce spray drift. J. Agric. Eng. Res., 67, 311-322.

Qi L., Miller P.C.H. \& Fu Z., 2008. The classification of the drift risk of sprays produced by spinning discs based on wind tunnel measurements. Biosyst. Eng., 100, 38-43.

Rioboo R., Voué M., Vaillant A. \& De Coninck J., 2008. Drop impact on porous superhydrophobic polymer surfaces. Langmuir, 24, 14074-14077.

Skuterud R., Nordby A. \& Tyldum A., 1988. Effects of application methods, spray volumes, pressures and herbicide rates on weed control in spring cereals. Crop Prot., 7, 303-308.

Solie J.B. \& Alimardani R., 1986. Predicting spray patterns of a vertical rotary disc atomizer. Trans. ASAE, 29(5), 1193-1198.

Southcombe E.S.E. et al., 1997. The international (BCPC) spray classification system including a drift potential factor. 
In: Proceedings of the 1997 Brighton crop protection conference - weeds, 17-20 November 1997, Brighton Centre and Stakis Brighton Metropole Hotel, Brighton, UK. British Crop Protection Enterprises, 371-380.

Taylor P., 2011. The wetting of leaf surfaces. Curr. Opin. Colloid Interface Sci., 16, 326-334.

Taylor W.A., 1981. Controlled droplet application of herbicides. Outlook Agric., 10, 333-336.

Van De Zande J.C., Holterman H.J. \& Porskamp H.A.J., 2002. Influence of reference nozzle choice on spray drift classification. In: Proceedings of the International advances in pesticide application: aspects of applied biology 66, 7-9 January 2002, University of Surrey, Guildford, UK. Warwick, UK: Association of Applied Biologists, 66, 49-55.
Walton W.H. \& Prewett W.C., 1949. The production of sprays and mists of uniform drop size by means of spinning disc type sprayers. Proc. Phys. Soc. London, Sect. B, 62, 341 .

Wolf T.M., Harrison S.K., Hall F.R. \& Cooper J., 2000. Optimizing postemergence herbicide deposition and efficacy through application variables in no-till systems . Weed Sci., 48, 761-768.

Zabkiewicz J.A., 2007. Spray formulation efficacy - holistic and futuristic perspectives. Crop Prot., 26, 312-319.

Zainoun N., Chicheportiche J.-M.\& Renaudeaux J.-P., 2004. Contrôle dimensionnel des aérosols par piézoélectricité. Gamac éditeur, 169-177.

(52 réf.) 